\title{
A Survey on different Image Deblurring Techniques
}

\author{
Fagun Vankawala \\ M. Tech (Computer \\ Engineering) (pursuing) \\ Charusat
}

\author{
Amit Ganatra, Ph.D \\ Department of Computer \\ Engineering \\ Charusat
}

\author{
Amit Patel \\ Charusat \\ CSTC Department
}

\begin{abstract}
Image blur is general artifacts in digital image processing and it is hard to avoid. Image enhancement or deblurring is necessary to reduce blur amount from the image. Image deblurring is a process used to reduce the blur quantity in a blurred image and make the degraded image into sharpened and clear image. When deblurring images, cause of blurring is very important to increase the effect of the deblurring to get good result. While working with real-time images, we may not have the knowledge of the reason of blurring. There are various sources why image gets blurred like motion blur, camera shake, out of focus blur, etc. This paper carried out performance comparison of different techniques to diminish the effects of above mentioned causes of blurring. The analysis and comparison was conceded out based on types of blur, Peak Signal-to-Noise Ratio (PSNR), Mean Square Error (MSE) and Execution time.
\end{abstract}

\section{Keywords}

Image Degradation, Image Deblurring, Deconvolution, Types of blur, Point Spread Function (PSF), Peak Signal-to-Noise Ratio (PSNR), Mean Square Error (MSE).

\section{INTRODUCTION}

Captured images are more or less blurry due to lot of interference in the environment and also in camera. The blurring caused by certain reasons like out-of-focus, motion of an object or camera, Gaussian blur, [1] etc. Recorded image has to be of a good quality. While using blurred image to get useful information for some applications, it is necessary to deblur the images. Image deblurring is used to make images sharp and retrieve as much as detailed information from the image. The image degradation equation is described as below [4]:

$$
\mathrm{g}=\mathrm{h} \otimes \mathrm{f}+\mathrm{n}
$$

Where, $\mathrm{g}$ is degraded image, $\mathrm{f}$ is original image, $\mathrm{h}$ is blur operator, $(\otimes)$ is the convolution process, $\mathrm{n}$ is additive. However, blur function $\mathrm{h}$ is unknown, it is essential to blur identification and blur estimation [7].

\subsection{Types of Blur:}

\subsubsection{Out of Focus}

It is blurring of an image due to incorrect focus. In optics, defocus image means an image is in out of focus. Detailed information in the image is blurred. Equation for this function is [3]:

$$
\mathrm{d}(\mathrm{x}, \mathrm{y})= \begin{cases}0 & \text { if } \sqrt[2]{x^{2}+y^{2}}>r \\ \frac{1}{\pi r^{2}} & \text { if } \sqrt[2]{x^{2}+y^{2}} \leq r\end{cases}
$$

Where, $\mathrm{R}$ is radius, according to radius PSF size will calculate i.e. for $\mathrm{r}, \mathrm{k}=2 \mathrm{r}+1$ and mask size $=\mathrm{k} \times \mathrm{k}$.

\subsubsection{Motion Blur}

It is blurring of an image due to movement of the object or imaging system [4]. It is the apparent fast moving objects in an image. There are two types of motion blur Linear that in a single direction and circular can be in angle.

\subsubsection{Box Blur}

It is a spatial domain linear filter in which each pixel in the resulting image has an equal value as the average value of its neighboring pixels in the input image.

\subsubsection{Gaussian Blur}

It is the result of blurring an image by a Gaussian function. It is a widely used in many graphics tools to reduce the details of an image. Equation for this function is [3]:

$$
G(x, y)=\frac{1}{2 \pi \sigma^{2}} e^{-\frac{x^{2}+y^{2}}{2 \sigma^{2}}}
$$

Where $\mathrm{x}$ is distance on the $\mathrm{x}$ axis from the origin, $\mathrm{y}$ is the distance on $y$ axis from the origin, and $\sigma$ is the standard deviation.

The overall methodology of this paper consists of retrieve enhanced image by using different deblurring algorithms like the iterative Richardson-Lucy, the iterative Van Cittert, the iterative Landweber, the iterative Poisson Map and Laplacian sharpening filters.

The structure of the paper is as followed. Section II describes narrative details of different deblurring algorithms. Section III demonstrates the comparison of described algorithms by experiments and simulation results. Section IV concludes the paper.

\section{DEBLURRING TECHNIQUES}

Image deblurring uses a point spread function (PSF) [8] to deconvolve the blurred image. Deconvolution is classified into two types: blind and non-blind deconvolution [1]. Blind deconvolution uses only blurred image for deblurring and in non-blind deconvolution use blurred image and known point spread function for deblurring. Blind deconvolution is more complex and more time consuming than the non-blind, because it estimates point spread function after each iteration [1]. This paper contains comparison of various non-blind algorithms like iterative Richardson-Lucy, the iterative Van Cittert, the iterative Landweber, and the iterative Poisson Map and also Laplacian sharpening filter.

\subsection{Iterative Richardson-Lucy Algorithm}

It is one of the most well-known deblurring algorithms for the many reasons like it doesn't depend on type of noise and it is also an iterative algorithm. The equation for this algorithm as below formula [4]:

$$
f^{n+1}=f^{n} \mathrm{H}\left(\frac{g}{H f^{n}}\right)
$$

Where, $f^{n+1}$ is the new approximation from the previous $f^{n}$, $g$ is the captured blurred image, $n$ is the number of the iterations, $\mathrm{H}$ is PSF and in the first iteration, $f^{n}$ is same as blurred image $g$. It minimizes the difference between the blurred image and the predicted image according to the Poisson statistics [4]. The drawback of this algorithm is the 
ringing effect and takes more time for more number of iterations.

\subsection{Iterative Van Cittert Algorithm}

It is an iterative algorithm for image deblurring. It decreases the difference between image obtained by subtracting the imaged estimate and the recorded image. The mathematical formula of this algorithm is below [5]:

$$
f^{n+1}=f^{n}+\left(\mathrm{g}-\mathrm{H} f^{n}\right)
$$

Where $f^{n+1}$ is the new approximation from the previous one $f^{n}, g$ is the captured blurred image, $n$ is the number of the iterations, $\mathrm{H}$ is PSF and in the first iteration, $f^{n}$ is same as blurred image $g$. One of advantage of this algorithm is that it contains simple mathematical operations. The limitations of it are sensitive to the noise in an image, unstable after some limit of iterations and the image can be look like shaky.

\subsection{Iterative Landweber Algorithm}

It is an enhanced version of Van Cittert algorithm. It is also an iterative algorithm. By using this algorithm, more stable and more reliable result can be achieved when performing an additional number of iterations. The equation of this algorithm is below [6]:

$$
f^{n+1}=f^{n}+\beta \mathrm{H}\left(\mathrm{g}-\mathrm{H} f^{n}\right)
$$

Where, $f^{\mathrm{n}+1}$ is the new approximation from the previous $f^{\mathrm{n}}, g$ is the blurred image, $n$ is the number of the iterations, $\mathrm{H}$ is the blur function called PSF, $\beta$ is a constant that controls the sharpening quantity, $f^{\mathrm{n}}$ in the first iteration same as blurred image $g$. The drawback of this algorithm is that it takes more time.

\subsection{Iterative Poisson Map Algorithm}

It is an iterative algorithm. It is same as Richardson-Lucy algorithm; the difference between these two algorithms is that, the Poisson Map uses a formula in the form of exponential. The equation of this algorithm is stated below [2]:

$$
f^{n+1}=f^{n} e^{\left[H\left(\frac{g}{H f^{n}}\right)-1\right]}
$$

Where, $f^{\mathrm{n}+1}$ is the new approximation from the previous $f^{\mathrm{n}}, g$ is the captured blurred image, $n$ is the number of the iterations, $\mathrm{H}$ is PSF. In the first iteration, the value of $\left(f^{\mathrm{n}}\right)$ is same as blurred image $g$. There are some limitations like complex computation because of using an exponential function and due to that reason it is slowest algorithm.

\subsection{Laplacian Sharpening Filters}

It is one of the well-known filters used for sharpening an image. Image sharpening is also a form of image deblurring. Laplacian filter is a $3 \times 3$ matrix which has three types, $-4,-8$ and 9 core matrixes. Types of Laplacian kernels are given below:

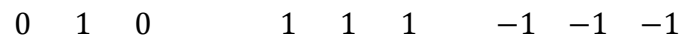

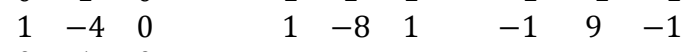

$$
\begin{aligned}
& \begin{array}{lllllllll}
0 & 1 & 0 & 1 & 1 & 1 & -1 & -1 & -1
\end{array}
\end{aligned}
$$

For this formula is [2]:

$$
\mathrm{F}=\mathrm{I}-[\mathrm{I} \otimes \mathrm{LK}]
$$

Where, $\mathrm{F}$ is the recovered image, $\mathrm{I}$ is the degraded image by Laplacian blur kernel and $\otimes$ is the convolution process. This is useful for image sharpening and takes less computation time. But it is not iterative.

\section{COMPARISON OF DIFFERENT NON- BLIND DEBLURRING ALGORITHMS}

This section includes a comparison between different deblurring techniques in terms of the mathematical operations, execution time, and image quality measures like PSNR, MSE.

In comparison of algorithms, two types of PSF were taken as Gaussian of $3 \times 3$ size and Disk for radius of 1 , iterations is taken as 2 for all algorithms but Laplacian is not an iterative so only one iteration result of Laplacian is compared. In Laplacian no of operations are only 3, PSNR for Gaussian blur is 23.2995 and execution time is 0.0910 seconds, where as for disk blur image PSNR is 25.4753 and execution time is

\begin{tabular}{|c|c|c|c|c|}
\hline PSF & Algorithm & $\begin{array}{c}\text { Mathematical } \\
\text { Operations }\end{array}$ & PSNR & $\begin{array}{c}\text { Execution } \\
\text { Time(s) }\end{array}$ \\
\hline \multirow{5}{*}{$\begin{array}{l}\text { Gaus } \\
\text { sian } \\
(3 \times 3)\end{array}$} & Blur & -- & 34.6665 & -- \\
\hline & $\begin{array}{c}\text { Iterative } \\
\text { Richardson- } \\
\text { Lucy }\end{array}$ & 4 & 41.3293 & 0.1424 \\
\hline & $\begin{array}{c}\text { Iterative } \\
\text { Van Cittert }\end{array}$ & 4 & 42.5365 & 0.0866 \\
\hline & $\begin{array}{c}\text { Iterative } \\
\text { Landweber }\end{array}$ & 5 & 42.2980 & 0.1190 \\
\hline & $\begin{array}{c}\text { Iterative } \\
\text { Poisson } \\
\text { Map }\end{array}$ & 6 & 41.8169 & 0.1168 \\
\hline \multirow{5}{*}{$\begin{array}{l}\text { Radi } \\
\text { us (1) }\end{array}$} & Blur & -- & 28.6338 & -- \\
\hline & $\begin{array}{c}\text { Iterative } \\
\text { Richardson- } \\
\text { Lucy }\end{array}$ & 4 & 30.1792 & 0.1207 \\
\hline & $\begin{array}{c}\text { Iterative } \\
\text { Van Cittert }\end{array}$ & 4 & 30.0598 & 0.0945 \\
\hline & $\begin{array}{c}\text { Iterative } \\
\text { Landweber }\end{array}$ & 5 & 30.0362 & 0.1320 \\
\hline & $\begin{array}{c}\text { Iterative } \\
\text { Poisson } \\
\text { Map }\end{array}$ & 6 & 27.8539 & 0.1219 \\
\hline
\end{tabular}
0.0953 seconds. Comparison of quality measures parameters for all other algorithms except Laplacian is shown in below table 1:

Table 1 Comparison of all algorithms

Simulation results are as shown in figure 1:

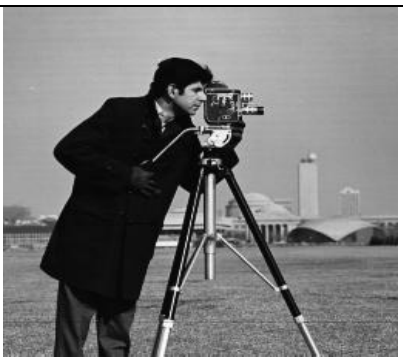




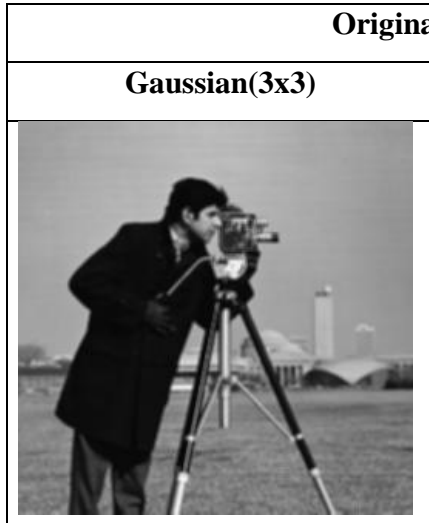

Blur

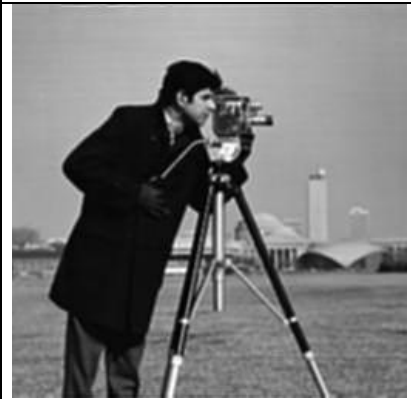

Iterative

Richardson-Lucy

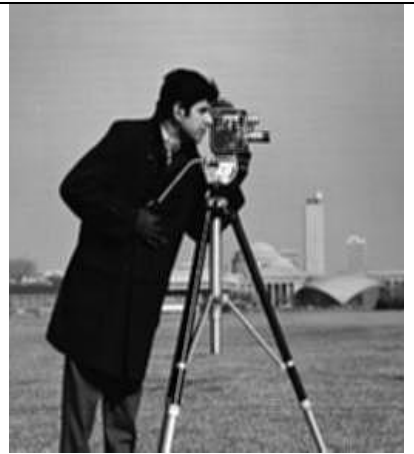

Iterative

Van Cittert

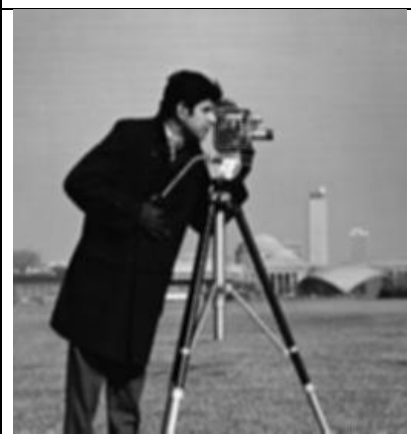

Iterative

Landweber
Radius(1)

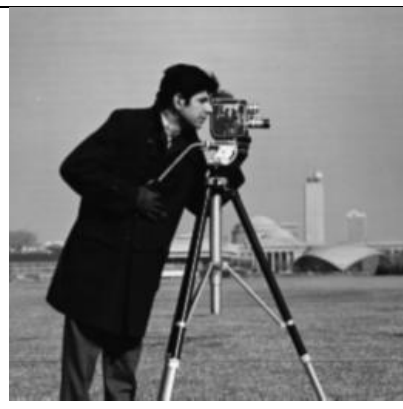

Blur

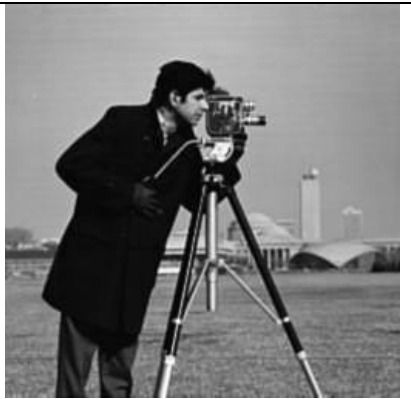

Iterative

Richardson-Lucy

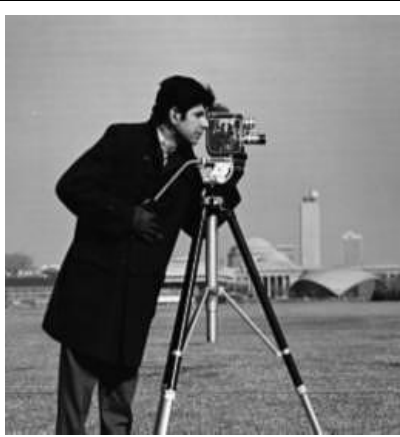

Iterative

Van Cittert

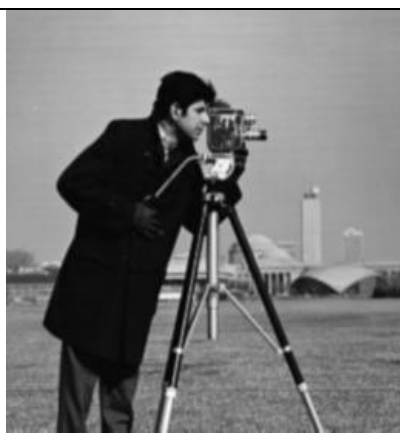

Iterative

Landweber

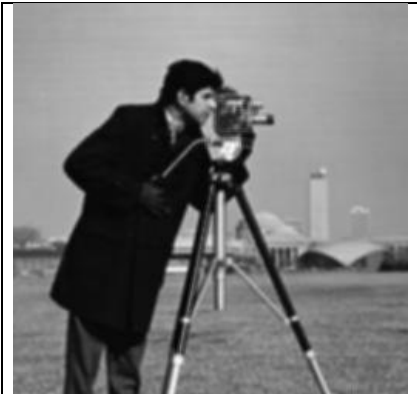

Iterative

Poisson Map

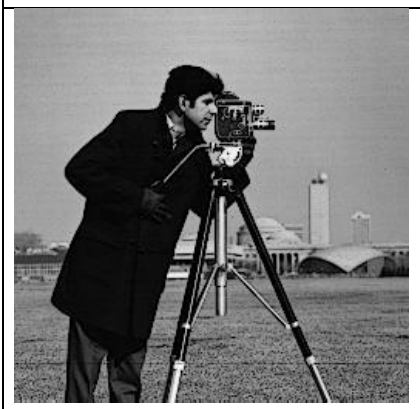

Laplacian Sharpening

Filters

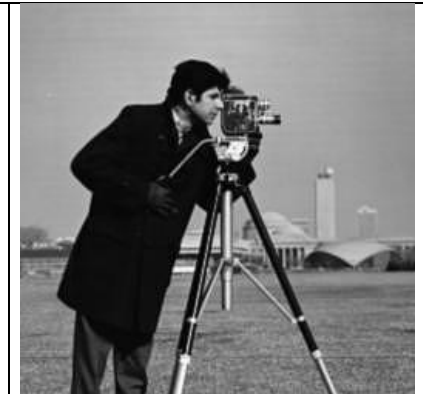

Iterative

Poisson Map

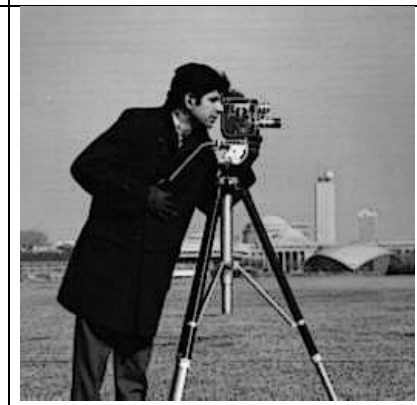

Laplacian Sharpening

Filters

Figure 1 Comparison based on simulations result

According to experiments, we can say that all algorithms are perform comparatively same and give same result in terms of PSNR and execution time but give better result if we known about the type of blur. If type of blur is unknown it is difficult to get better result. Therefore, we can use Blind Convolution but one drawback of it takes much time to calculate PSF for convolution according to blur. Performance of these algorithms was tested for an image with noise present in it. To carry out this analysis, an image "cameraman" was blurred using Gaussian smoothing and Gaussian noise function was applied to the image. For different number of iterations all algorithms perform differently i.e. some give better result in less number of iteration whereas to get better result perform more number of iterations of some of them. Table 2 shown below depicts performance of different deblurring algorithm in presence of noise.

Table 2 Performance comparison in presence of noise

\begin{tabular}{|c|c|c|}
\hline Algorithm & $\begin{array}{c}\text { Time } \\
(\mathbf{s e c})\end{array}$ & PSNR \\
\hline Blur & - & 19.9864 \\
\hline Richardson-Lucy & 0.3341 & 17.7841 \\
\hline Van Cittert & 0.1259 & 17.2201 \\
\hline Landweber & 0.1303 & 17.0474 \\
\hline Poisson Map & 0.1372 & 18.6088 \\
\hline
\end{tabular}

According to above table experiments show that all are performs poorly in the presence of noise. 


\section{CONCLUSION}

Based on the literature study and simulation results analysis on various image deblurring algorithms proposed by different researchers, conclusion can be made that for different algorithms Gaussian as PSF, Van Cittert and Disk as PSF, Richardson Lucy gives comparatively better results and also observed that Richardson-Lucy and Van Cittert have same number of operations (4) where as Poisson Map has highest number of operations (6) and Laplacian has lowest number of iterations (3). Moreover, also conclusion can be made that Laplacian gives poor result because in this for convolution on image use PSF which described in section 2.5 instead of using PSF according to blur. Based on the study carried out on a noisy image, it can be concluded that these algorithms perform poorly in presence of noise in an image. Therefore, to get better result with noisy image will be the main task for future work.

\section{REFERENCES}

[1] Rohina Ansari,Himanshu Yadav,Anurag Jain," A Survey on Blurred Images with Restoration and Transformation Techniques", International Journal of Computer Applications (0975 - 8887) Volume 68- No.22, April 2013

[2] Zohair Al-Ameen, Ghazali Sulong and Md. Gapar Md. Johar," A Comprehensive Study on Fast image Deblurring Techniques", International Journal of Advanced Science and Technology Vol. 44, July, 2012

[3] Dejee Singh1,Mr R. K. Sahu, "A Survey on Various Image Deblurring Techniques", International Journal of Advanced Research in Computer and Communication Engineering Vol. 2, Issue 12, December 2013

[4] Jiunn-Lin Wu, Chia-Feng Chang, Chun-Shih Chen," An Improved Richardson-Lucy Algorithm for Single Image Deblurring Using Local Extrema Filtering", IEEE International Symposium on Intelligent Signal Processing and Communication Systems (ISPACS 2012) November 4-7, 2012

[5] A. Bennia and S. M. Riad, "Filtering Capabilities and Convergence of the Van-Cittert Deconvolution Technique", IEEE Transactions on Instrumentation and Measurement, vol. 41, no. 2, 1992

[6] L. Lang and Y. Xu, "Adaptive Landweber method to deblur images", IEEE Signal Processing Letters, vol. 10, no. 5,2003

[7] Hanghang Tong,Mingjing Li, Hongjiang Zhang,Changshui Zhang," Blur Detection for Digital Images Using Wavelet Transform*,"Multimedia and Expo, 2004. ICME '04. 2004 IEEE International Conference on (Volume: 1 ).

[8] Xue-fen Wan, Yi Yang, Xin Lin," Point Spread Function Estimation For Noisy Out-of-focus Blur Image Restoration", IEEE International Conference on Software Engineering and Service Sciences (ICSESS), 2010

[9] Ashwini M. Deshpande, Suprava Patnaik "Comparative Study and Qualitative-Quantitative Investigations of Several Motion Deblurring Algorithms", 2nd International Conference and workshop on Emerging Trends in Technology (ICWET) 2011 Proceedings published by International Journal of Computer Applications

[10] Rupali Yashwant Landge, Rakesh Sharma," Blur Detection Methods for Digital Images-A Survey", International Journal of Computer Applications Technology and Research Volume 2- Issue 4, 495 - 498, 2013

[11] Dejee Singh1 , Mr R. K. Sahu "A Survey on Various Image Deblurring Techniques", International Journal of Advanced Research in Computer and Communication Engineering Vol. 2, Issue 12, December 2013 